\section{Solution des délais: doit-on, peut-on prendre position en tant que médecin?}

Ursula Steiner-König, vice-présidente de la FMH

\section{Panta rhei: tout est mouvement}

Il n'y a encore qu'un demi-siècle, il était de règle qu'une seule norme morale règne au sein de la famille, de l'école et de l'église, comme si l'ancrage philosophico-religieux pouvait faire barrage contre le désespoir. Aujourd'hui au contraire, la notion du "tout est mouvement" est de plus en plus de mise dans notre société multiculturelle et pluraliste et tout consensus sur les questions morales devient caduc. Cette perte de repères dans la société actuelle pose l'urgence d'une réflexion éthique et de directives en la matière. Si nous devions néanmoins attendre de l'éthique en général qu'elle nous fournisse des instructions marquées au sceau de la rigueur mathématique, ce serait nous tromper lourdement, comme si nous voulions la réduire à des sentiments et à des émotions invérifiables. Selon A. Bondolfi [1], il nous faut une "voie médiane qui permette au discours éthique de présenter une certaine vraisemblance entre simple opinion et réalité sans artifices".

Je me permets ci-après - me fondant sur une sélection de réflexions éthiques - d'exprimer quelques idées sur le thème de la grossesse et de son interruption.

Dans notre univers culturel du moins, le respect de la personnalité, de la volonté et des droits de la personne a gagné en importance. L'éthique médicale et son application à l'éthique de l'acte médical sur l'individu malade [1] «n'est pas simplement un acte rationnel; il inclut la dimension de la communication", qui trouve précisément toute son importance dans la relation médecin - patient. Le côté pratique n'est pas pour autant négligé. La science médicale allie donc l'observation des sciences naturelles et l'intervention fondée sur l'éthique.

Selon le serment d'Hippocrate, l'acte médical doit suivre le principe du «primum non nocere»: d'abord ne pas nuire. Les conflits éthiques impliquent le cas échéant, plus souvent qu'autrefois, des domaines médicaux et non-médicaux. L'équilibre entre les éléments en cause ne concerne pas uniquement le domaine de la science médicale, mais aussi l'opinion du patient. A cet égard, on n'oubliera pas que ce dernier se trouve - psychologiquement parlant - dans une situation de dépendance. Les notions d'autonomie, détournement des maux, d'assistance et de justice [2] ont aujourd'hui valeur de principes reconnus de l'acte médico-éthique, aussi bien dans les pays anglosaxons que dans l'espace culturel européen.

En parallèle de cette vision des choses, on voit s'accroître la tendance à tout vouloir objectiver et légaliser.

Concernant la tendance à objectiver, je dirai que le but à atteindre est de tout vouloir maîtriser - sans tache aveugle ni chambre noire - et de comprendre l'ensemble du domaine des sciences naturelles, ce qui permettrait d'en subdiviser les éléments dans toute leur complexité.

Il convient d'ajouter qu'en faisant la différence entre corps biologique et corps habité (Körper-Leib), l'impondérable, le secret, le merveilleux de la vie humaine et de son développement in utero est voué à être tenu en haute estime (voir plus loin).

A mon avis, ce serait une tentation risquée que de croire à une possibilité d'objectivation pleine et entière. Dans notre société pluraliste, précisément, qui voit se délabrer les valeurs traditionnelles ou religieuses, voire les deux, notre profession devrait se prononcer clairement, face au public, pour la reconnaissance des secrets de la vie en dépit des avancées médicales et des progrès techniques. Il suffit de songer au problème de la totipotence des cellules-souche prélevées sur les embryons!

0. Guillod [3] déclare que le droit, par rapport aux intérêts conflictuels en présence, vise clairement des décisions définitives, applicables pour tous. Dans le domaine de l'activité médicale, citons les lois fédérales sur l'exercice de la profession médicale, sur l'assurance-maladie, le Code des obligations et les lois sanitaires cantonales (sans compter de nombreux arrêts de droit). Le droit se fonde sur une image de l'homme libre, responsable, doté d'une dignité intrinsèque, ainsi que sur des valeurs (principes de justice, de loyauté et de refus de nuire) dont il assure la protection et l'encouragement. 0 . Guillod termine son exposé [3] en soulignant que "la réglementation des pratiques médicales nous pousse aux limites du droit, de la déontologie et de l'éthique». La condition d'une entente est de demander que "chacun tente de comprendre la langue de l'autre, sa manière de penser, en un mot d'adopter une position d'interdisciplinarité».

A mon avis, la notion de "consentement éclairé» se situe précisément dans cette zone interdisciplinaire et fera plus loin l'objet d'une observation plus précise, notamment par rapport au cas de la femme enceinte involontairement. Disons toutefois que la notion même de consentement éclairé exige un dialogue entre celui qui demande de l'aide et celui qui aide. Ces rapports humains conduisent à voir la personne non seulement sous son aspect purement objectif, mais sous son aspect de sujet, et cette reconstruction garantit que la médecine détient et conserve son caractère relationnel. [4] Dans cette optique, l'éthique du médecin et l'éthique du patient ont la même valeur. 


\section{Responsabilité en tant que principe éthique de base}

Dans notre civilisation, le principe de responsabilité - en analogie avec le principe d'autonomie - a pris une importance morale grandissante. R. Spaemann donne sa version du principe de responsabilité: "on ne saurait parler de responsabilité lorsqu'il s'agit de suivre des directives précises sur la manière d'agir, mais bien plutôt lorsqu'un responsable est désigné pour établir des règles dans un domaine vital complexe ou chargé d'exécuter une tâche particulièrement difficile avec une marge d'appréciation et pour le résultat de laquelle il est responsable. Dans des situations complexes, l'être humain doit révéler de la souplesse et de l'aptitude au changement. Changer n'est cependant possible que pour celui qui en a la compétence. A cela s'ajoute que de plus en plus de personnes ont plusieurs rôles à leur actif" [5]. Les rôles exigent des comportements définis. La coordination de plusieurs rôles demande des décisions dépassant le comportement préétabli.

Si la technique moderne a permis à maints égards plus de transparence et d'efficacité dans le domaine de l'activité humaine, l'apport de connaissances ainsi acquises conduit cependant à plus de responsabilités. Les conditions de vie ayant aussi tendance à rapidement changer, il sera de plus en plus difficile de se fonder sur une stratégie d'action plus ou moins définie. Aussi bien du côté du corps médical que des femmes concernées, il convient de connaître la marge d'appréciation précitée, ainsi que l'obligation de rendre compte qui lui est liée

Les principes éthiques (autonomie, assistance, justice) sont aujourd'hui débattus dans une approche éthique faite de vertu. On citera brièvement $R$. Spaemann [5] qui dit de la responsabilité qu'«elle est toujours issue de situations dans lesquelles nous nous trouvons, à savoir morales. Les situations où la morale joue un rôle sont les suivantes: amitié, mariage, relation parents-enfants, médecin-patient, enseignants-élèves, ainsi que les relations entre collègues. La réalisation de ces principes passe néanmoins par une attitude à adopter, en adéquation ciblée à la morale, sans égoïsme, passion ou fanatisme."

Si nous tentons d'appliquer ces principes à la réglementation des délais, force est de reconnaître que nous nous trouvons devant une situation complexe, que les situations individuelles jouent un rôle important et qu'aussi bien le médecin que la femme concernée peuvent revendiquer des options différentes.

\section{Evaluation des intérêts}

Le médecin fera bien, en relation avec le problème posé, d'observer que par rapport au risque pour la santé que peut représenter une grossesse, il lui faudra prendre en compte non seulement la dimension physique, mais aussi psychique et sociale du cas en question. Le fait d'être enceinte signifie non seulement qu'il faut donner à l'embryon les conditions de son développement somatique, mais qu'hormis le côté biologique, il s'agit aussi de prévoir les conditions optimales du développement d'un être en devenir sur le plan psychique et social. Si la femme enceinte ne s'en sent pas capable, c'est au médecin de l'accueillir. Si celui-ci tient compte de l'embryon engendré, la situation se complique du fait que l'embryon n'a pas de réel intérêt mais uniquement un intérêt moral. [5]

On entend souvent, dans le corps médical, que les femmes involontairement enceintes sont sous la pression de tiers et ne peuvent donc faire valoir leur propre opinion. C'est dès lors au médecin consulté d'éclaircir la situation avec la personne concernée. Il peut arriver que la femme concernée - le cas peut fort bien se présenter - accepte enfin sa grossesse et qu'un délai de réflexion lui permette de prendre sa décision en toute connaissance de cause. Il est possible aussi que le fardeau représenté par une grossesse non désirée demeure trop lourd. La volonté d'avorter viendra alors réellement de l'intéressée.

H. M. Sass, éthicien [6] voit dans l'éthique médicale se dessiner une "ère copernicienne", dans la mesure où la responsabilité personnelle du patient remplace les classiques maximes hippocratiques du «nihil nocere" (ne pas nuire) et du «bonum facere» (faire le bien). Le pouvoir d'appréciation et de décision ne relève plus de la (presque) seule responsabilité du médecin, mais de celle du médecin et du patient. Le médecin, quant à lui, devra se pencher sur les problèmes suivants: approche des facteurs de risque individuels et spécifiques aux groupes, développement de stratégies en vue de diminuer ou de prévenir les risques individuels pour la santé et de réaliser des objectifs sanitaires individuels et de société.

Face à une grossesse non souhaitée ou refusée, le médecin se trouve confronté à deux types de situation: d'une part, il doit comprendre le problème sous l'angle psychodynamique, donc avoir une approche empathique et critique de la surcharge émotionnelle et de l'impasse dans laquelle se trouve l'intéressée, afin de juger si les ressources de celle-ci sur le plan personnel et psychique lui permettent ou non de supporter une grossesse. D'autre part, il doit prendre en compte la réalisation pratique de l'interruption de grossesse. Selon la loi en vigueur, ces deux éléments du problème sont clairement distincts, puisqu'il faut l'accord de deux confrères, le plus souvent un gynécologue ou un généraliste, ainsi qu'un psychiatre. Pour une fois, la tâche semble souvent relativement plus facile dans le domaine de la psychiatrie que dans celui de la chirurgie, les tenants du premier domaine n'étant pas confrontés avec le fœtus avorté. Du moins, lorsqu'il ne s'interdit pas sa résonance intérieure, le chirurgien peut être confronté à un conflit de conscience plus aigu que le psychiatre. De nombreux témoignages sont là pour le confirmer.

Dans un article rédigé sur le sujet, Judith Pòk relève que pour elle en tant que gynécologue, l'embryon et la mère, sur le plan de leur existence biologique, ne sauraient être séparés l'un de l'autre. Cette manière de voir les choses ne me semble pas seulement digne de réflexion sur le plan somato-physio- 
logique, mais elle illumine un tout autre aspect, juridique et éthique: selon la Convention européenne des droits de l'homme et de la biomédecine, seule compte la décision de la personne concernée, les intérêts de tiers ne sauraient être pris en compte (cet argument a été notamment avancé dans la foulée des travaux préliminaires d'une législation sur la stérilisation, lorsqu'on a parlé du droit personnel absolu d'une personne incapable de discernement de mener à terme sa grossesse: ni les intérêts de l'enfant non-né, ni ceux des grands-parents potentiels ne pouvaient être pris en compte!). Si l'on place cet argument en parallèle d'une vision "holistique» d'une indivisibilité de l'être, il en ressort nettement qu'au stade précoce de la grossesse, l'embryon n'a pas encore de droit légal particulier. La question n'est donc pas de savoir à partir de quel moment il serait détenteur de ce droit lui accordant simultanément la protection, mais de savoir à partir de quel moment il devient un fotus pouvant vivre indépendamment de sa mère, à savoir un individu. Je pense dès lors qu'il faut accorder la priorité à la femme involontairement enceinte de donner son propre avis, selon son éthique personnelle, sur la situation conflictuelle du pour ou du contre en matière d'avortement. C'est rendre mieux hommage, me semble-t-il, à la complexité de la situation: il ne s'agit pas, en effet, de savoir à partir de quand un embryon - sur le plan scientifique également - doit ou peut obtenir le statut d'"être humain", mais uniquement de reconnaître cette "unité holistique» du début d'une nouvelle vie in utero.

Il est inacceptable d'attribuer au corps médical l'intention - si la solution des délais entrait en vigueur - d'accepter de procéder à des avortements jusqu'à peu de temps avant l'accouchement, équivalent à un déni de respect de la vie humaine.

\section{Ce corps que j'ai, cet être qui est moi}

En Suisse également, on reconnaît de manière générale que le souhait de vouloir un enfant peut et doit relever de la liberté individuelle de décision et de planification que l'on donne à sa vie. L'événement constitué par la procréation, la grossesse et la naissance est ressenti par la femme, de par son ambiguïté et son potentiel conflictuel, de manière plus intense et plus consciente que par le passé.

La femme tombée involontairement enceinte ressent l'événement comme radical et porteur de conséquences graves, déchirée qu'elle est par un conflit inéluctable entre "le corps que j'ai et l'être que je suis» [7]. Sa raison rejetait ce qui lui advenait sous forme de réalité tangible (R. Spaemann fait allusion à plusieurs reprises au "genitum non factum" du credo chrétien [5]. Cette nouveauté de la grossesse au stade initial conduit précisément - si l'on examine attentivement le conflit de la femme en question - à un dilemme considérable et, le cas échéant, à des tensions décisionnelles importantes. Honte, peur, sentiment d'échec, de culpabilité, de désécurisation et autres facteurs émotionnels peuvent conduire à rendre im- possible la mise à jour de la situation conflictuelle par l'intéressée. On ne saurait non plus exclure, en l'occurrence, un refoulement total de ce qui touche véritablement au vif. Il s'ensuit une scission de l'être au profit d'une perception réductionniste du corps. S'agissant de "consentement éclairé», tous ces éléments doivent être pris en compte (pourquoi dit-on en fait "sage-femme»? De quelle sagesse pourrait-il être question?).

Si la situation se présente bien, la femme enceinte consulte à temps. Dans le cas contraire, elle peut dépasser le moment où l'avortement est permis. Ces réflexions ont conduit à un long et profond débat dans les milieux politiques sur l'obligation de conseiller. L'importance serait dès lors accordée au rôle de l'Etat dans la protection de la vie humaine. Si l'on veut, ici aussi, se référer à une vision holistique des choses, il faudrait (on pourrait) répéter que le devoir de protection se rapporte à un seul corps, celui de la mère, dont l'embryon précoce fait partie.

\section{Ethique du citoyen - éthique du médecin}

Les notions de corps, de réalité corporelle et de réalité de l'être (Leib, Leibhaftigkeit, Leiblichkeit) nous permettent de prendre une distance par rapport à la scission somato-psychique. Je suis tentée de faire le rapprochement avec la notion de "médecin" (Arzt) qui diffère, sur le plan de la tonalité et de son importance, de celle de "thérapeute" (Mediziner). A mes yeux, le médecin voit son vis-à-vis comme un tout, alors que le soignant voit principalement la pathologie dont il souffre. Une troisième notion, voisine dans ce contexte, serait celle de "salut».

Une "troisième voie" serait-elle donc possible dans l'approche de notre difficile objet de réflexion? Peut-on résoudre ainsi le problème de la contradiction entre protection absolue de la vie humaine (impliquant le devoir de mener sa grossesse à terme), et avortement mené, semble-t-il, sans émotion et finalement de manière immorale et illégale? Y a-t-il une solution d'éthique médicale qui puisse ouvrir au médecin - tenaillé par les conflits de croyance ou de conscience (comme d'ailleurs tout citoyen) - une vision plus large du conflit que pourrait générer la réglementation des délais?

Précisément au moment d'écrire ces lignes, je lis dans la NZZ du 3 et 4 novembre 2001 qu'un procès pénal s'est tenu au Portugal dans une halle de tennis, faute de place au tribunal, contre 43 hommes et femmes (l'accusée principale était une sage-femme ayant tenu une "clinique» illégale pour avortements); 17 femmes avaient souhaité avorter et parmi les "co-auteurs", on comptait un médecin, un chauffeur de taxi, des aides soignants et de pharmacie ainsi que des travailleurs sociaux. Devons-nous accepter, médecins de ce pays, d'être à la merci de tels procès potentiels? Les femmes en détresse doivent-elles continuer à être taxées de criminelles?

Le philosophe H. G. Gadamer nous donne sa formule à réflexion selon laquelle «vouloir «rationaliser 
toutes les formes de vie et domaines y attenant conduit à une situation évoluant ıde plus en plus vers la forme panique d'une fuite devant la libertém [8]. On ne s'étonnera pas non plus qu'il parle ailleurs d' «assistance libératrice» de la part du médecin [8]. En effet, Gadamer relève que «la santé n'est pas ce qui est visible lors de l'examen médical, mais une notion abstraite qui se montre tout en étant absente. Nous n'abordons donc pas la santé de manière consciente et elle ne nous préoccupe pas comme la maladie. Ce n'est pas quelque chose qui nous invite à nous soigner ou à nous alarmer en permanence. Elle relève du miracle qui est celui de l'oubli.» (Wunder der Selbstvergessenheit). Il évoque aussi le clair-obscur (Verborgenheit) que constitue la santé et déclare que «la santé se maintient elle-même dans ses propres valeurs, les valeurs courantes que l'on acquiert par l'expérience et qui se fonde sur le cas d'espèce, de manière à éviter l'inadéquation». Et il explique la notion d'adéquation: "Si la santé ne saurait se mesurer réellement, c'est parce qu'elle est un état d'adéquation intérieure, de correspondance avec soi-même, que l'on ne saurait remplacer par un autre contrôle. Dès lors, la question au patient demeure pertinente de savoir s'il se sent malade». En conformité avec cette adéquation intérieure qui, chez les Grecs, était «harmonie, adéquation mesurable", le médecin devrait, hormis le mesurable et l'objectivable, considérer qu'il ne s'agit pas uniquement de traiter, mais de guérir ou, concernant la femme enceinte sans l'avoir voulu, de conseiller.

Aucun médecin ne souhaite, en fin de compte, se fermer à la détresse d'autrui. En tant que citoyens et citoyennes de ce pays, nous sommes appelés à voter sur un sujet qui mérite à bon escient le plus grand des respects. Nous ne saurions vouloir, en effet, être accusés de nous substituer à la puissance divine ni faire usage d'un droit de vie ou de mort. Au nom de l'assistance libératrice, nous oserions cependant peser l'opinion du médecin et le devoir du citoyen. Se décider devant le conflit intérieur entre objectivité et subjectivité/individualité de la situation revient à dire que l'on ne saurait pas simplement accepter, en tant que médecin, une éventuelle déshumanisation de l'image actuelle de l'être humain en tant que processus inévitable. Je suis prête à reconnaître les arguments des opposants à la solution des délais, précisément parce que je refuse toute généralisation de l'humain et toute intention d'objectiver celui-ci. 0. Guillod, dans son article «Droit, éthique et médecine - harmonie ou dissonnances?» déclare: «Dans le domaine du droit et de l'éthique, force est de constater aujourd'hui une convergence - si ce n'est un amal- game. La question se pose de savoir si le domaine de la biomédecine ne serait pas le champ d'expérimentation de nouvelles normes (juridiques, parajuridiques, métajuridiques), destinées à accompagner des pratiques en cours. Fixer des conditions générales relève, à mon avis, du droit, ce qui implique le devoir de fixer des limites (comme le cadre d'un tableau) données par des valeurs fondamentales (reconnues par l'éthique comme par le droit), telles que la protection de la vie et de la santé de l'être humain, ainsi que la promotion de l'autonomie. Mais fixer des limites permet aussi de garantir la sécurité du droit et de créer des zones de liberté au sein du cadre. Seuls les chefs-d'œuvre méritent un cadre pour les mettre en valeur. L'idéal serait d'en avoir un aussi pour l'accompagnement juridique de la pratique médicale.» [3]

\section{Conclusions}

"Panta rhei»: si tout est mouvement, la décision des urnes répondra à "l'état de l'erreur le plus actuel» (neuster Stand des Irrtums). L'important est de ne pas nous fermer aux changements de société. Simultanément, le «miracle de la vie» doit continuer à inspirer à notre vocation le plus grand des respects.

\section{Références}

1 Bondolfi A. Neuere Tendenzen und grundsätzliche Überlegungen zum Verhältnis von Medizin und Ethik. Dans: Bondolfi A, Müller H (éds.). Medizinische Ethik im Alltag. Bâle: EMH Editions médicales suisses SA; 1999. p. 17-38.

2 Bondolfi A. Die moralischen Prinzipien ethischen Handelns. Dans: Bondolfi A, Müller H (éds.). Medizinische Ethik im Alltag. Bâle: EMH Editions médicales suisses SA; 1999. p. 83-122.

3 Guillod 0. Recht, Ethik und Medizin: Harmonie oder Dissonanzen? Dans: Bondolfi A, Müller H (éds.). Medizinische Ethik im Alltag. Bâle: EMH Editions médicales suisses SA; 1999. p. 63-82.

4 Kress JJ. Die Sicht eines Psychiaters. Dans: Tröhler U, Reiter-Theil S. Ethik und Medizin, 1947 à 1997, Was leistet die Kodifizierung von Ethik? Göttingen: Wallstein Verlag; 1997. p.513-5.

5 Spaemann R. Grenzen. Zur ethischen Dimension des Handelns. Stuttgart: Klett-Cotta; 2000

6 Sass H-M. Medizinethik. Dans: Pieper A, Thurnherr U. Angewandte Ethik. Beck'sche Reihe BsR. 1261. Munich: C. H. Beck; 1998. p. 80-109.

7 Graf Dürckheim K. Vom doppelten Ursprung des Menschen. Herderbücherei, vol. 911. $7^{\mathrm{e}}$ édition. Fribourg en Brisgau: Herder; 1983.

8 Gadamer HG. Über die Verborgenheit der Gesundheit. Bibliothek Suhrkamp. Frankfort sur le Main: Suhrkamp; 1993. 